\title{
Foot Digit 2 Phalanx
}

National Cancer Institute

\section{Source}

National Cancer Institute. Foot Digit 2 Phalanx. NCI Thesaurus. Code C52779.

A bone in the second toe from the medial side of the foot. 